\title{
3D dictionary learning based iterative cone beam CT reconstruction
}

\author{
Ti Bai, Hao Yan, Feng Shi, Xun Jia, Yifei Lou, Qiong Xu, Steve Jiang, Xuanqin Mou \\ Department of Radiation Oncology, UT Southwestern Medical Center, Dallas, TX, USA.
}

Received March 19, 2014; Published Online April 08, 2014

[Presented at the Young Investigator's Symposium at the 2014 Annual Meeting of

Southwest Chapter of American Association of Physicists in Medicine (AAPM) in San Antonio, Texas, USA]

\section{Conference Proceeding}

\begin{abstract}
Purpose: This work is to develop a 3D dictionary learning based cone beam CT (CBCT) reconstruction algorithm on graphic processing units (GPU) to improve the quality of sparse-view CBCT reconstruction with high efficiency.

Methods: A 3D dictionary containing 256 small volumes (atoms) of $3 \times 3 \times 3$ was trained from a large number of blocks extracted from a high quality volume image. On the basis, we utilized cholesky decomposition based orthogonal matching pursuit algorithm to find the sparse representation of each block. To accelerate the time-consuming sparse coding in the $3 \mathrm{D}$ case, we implemented the sparse coding in a parallel fashion by taking advantage of the tremendous computational power of GPU. Conjugate gradient least square algorithm was adopted to minimize the data fidelity term. Evaluations are performed based on a head-neck patient case. FDK reconstruction with full dataset of 364 projections is used as the reference. We compared the proposed 3D dictionary learning based method with tight frame (TF) by performing reconstructions on a subset data of 121 projections.
\end{abstract}

Results: Compared to TF based CBCT reconstruction that shows good overall performance, our experiments indicated that $3 \mathrm{D}$ dictionary learning based CBCT reconstruction is able to recover finer structures, remove more streaking artifacts and also induce less blocky artifacts.

Presenting author: Ti Bai; Department of Radiation Oncology, UT Southwestern Medical Center, Dallas, TX, USA.

Cite this article as:

Bai T, Yan H, Shi F, Jia X, Lou Y, Xu Q Jiang S, Mou X. 3D dic-

tionary learning based iterative cone beam CT reconstruction. Int J Cancer Ther Oncol 2014; 2(2):020240.

DOI: $10.14319 / \mathrm{ijcto} .0202 .40$
Conclusion: 3D dictionary learning based CBCT reconstruction algorithm is able to sense the structural information while suppress the noise, and hence to achieve high quality reconstruction under the case of sparse view. The GPU realization of the whole algorithm offers a significant efficiency enhancement, making this algorithm more feasible for potential clinical application.

Innovation/Impact: In this work, we proposed a sparse view cone beam CT reconstruction algorithm based on 3D dictionary learning which contained stereo structures adaptive to CT volume. Moreover, we utilize GPU to accelerate the sparse coding process which is very time-consuming due to the large amount of blocks.

Key Results: We performed a sparse view CBCT reconstruction on a head neck data consists of 364 projections to validate our algorithm. Figure 1 displays the reconstruction results using different algorithms. Figure $1(\mathrm{a})$ is the reference image reconstructed by FDK utilizing all the projections. Figure $1(\mathrm{~b})$ and (c) are the tight-frame (TF) and 3D dictionary learning based methods using 121 projections, respectively. It is shown that $3 \mathrm{D}$ dictionary learning based reconstruction outperforms TF based one, for example, it could keep structure in a finer manner, removes more noise/streaking artifacts, while introduce less blocky artifacts. 


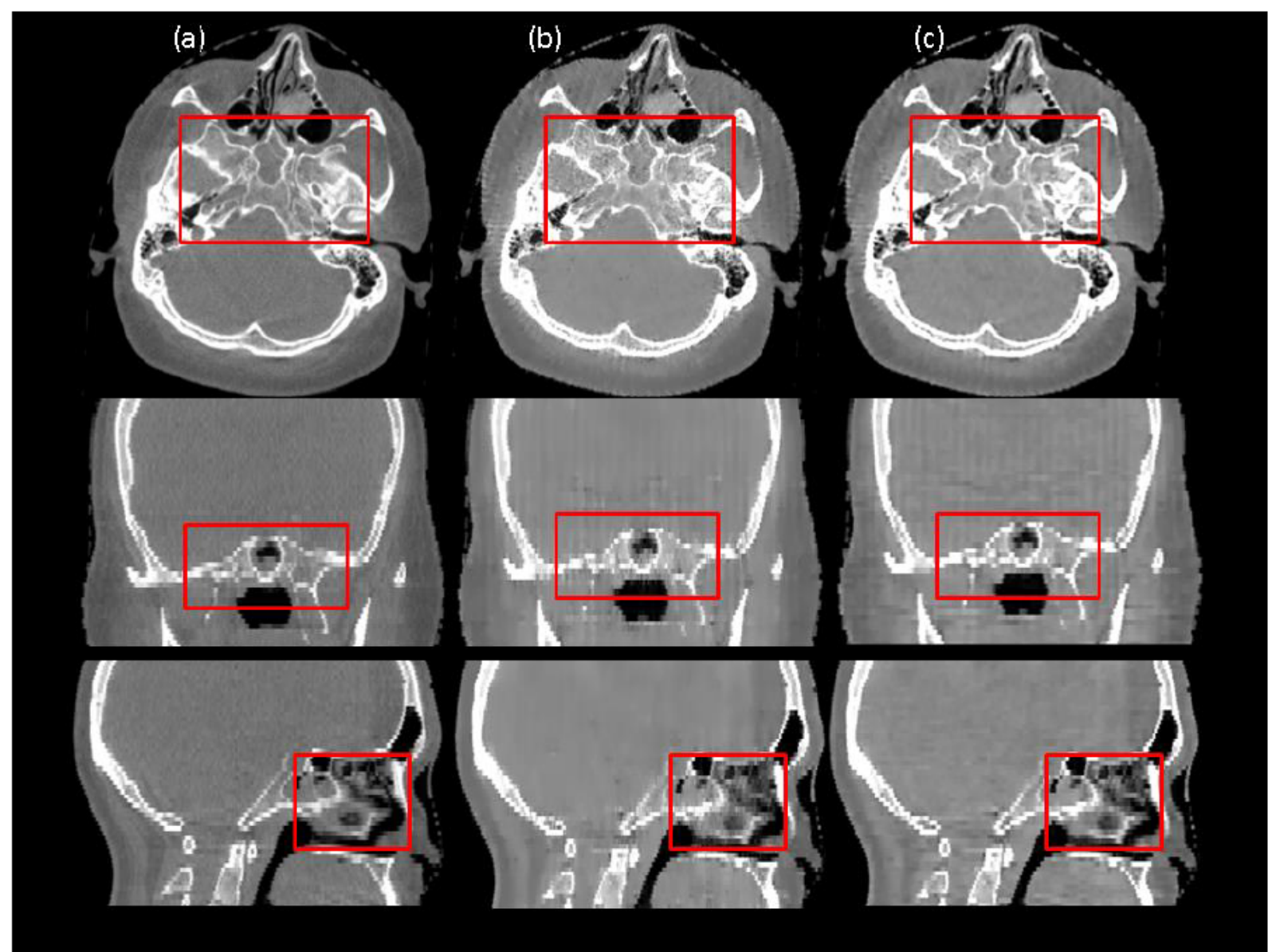

FIG. 1: Reconstructions of different planes by using different number of data and different algorithm. From top to bottom, the images correspond to transverse plane, coronal plane and sagittal plane. The first column is reconstructed by FDK using all the 364 projections, while the second column and the third column are reconstructed by TF based and 3D dictionary learning based algorithm using 121 projections, respectively. 